\title{
The genetic diversity of Paeonia anomala (Paeoniaceae), as indicated by nuclear ITS and plastid ycf1 molecular markers
}

\author{
Galina Degtjareva*, and Sergey Efimov \\ Botanical Garden, Faculty of Biology, M.V. Lomonosov Moscow State University, 119991, Moscow, \\ Russia
}

\begin{abstract}
Variability of nuclear ITS rDNA and plastid ycf1 gene was studied to elucidate genetic diversity of Paeonia anomala (Paeoniaceae), which possesses the most extensive range among all species of the genus. Sixteen populations were sampled across the entire distribution range of the species. The principal coordinate analysis suggested the existence of three ycf1 haplotype groups distributed in distinct geographical locations. For ITS region, no variability throughout the range was found; only samples of $P$. anomala from its north-western range margin (the Kola Peninsula) are characterized by unique nucleotide substitutions.
\end{abstract}

\section{Introduction}

The genus Paeonia L. comprises about 35 species of shrubs and perennial herbaceous plants that are common in the northern hemisphere. The genus is well distinguished phylogenetically, being the only representative of the family Paeoniaceae. However, the relationships between the species are rather confusing. The situation is partly complicated by the fact that hybridization processes took place in the formation of species complexes, which were also confirmed by molecular methods [1-2].

In the present study, we used nuclear ITS rDNA and plastid ycf1 gene to investigate the phylogeographic pattern of the perennial herb Paeonia anomala. The species occupies a wide continental distributional range, disjunctly extending from northern Europe to central Siberia, Mongolia and China [3]. Throughout its distribution range, Paeonia anomala shows a wide phenotypic variation, both in reproductive and vegetative traits, with the description of subspecies or varieties [4].

Our previous study based on nuclear ITS rDNA and plastid ycf1 gene showed that $P$. anomala represents a separate species, distinct from $P$. hybrida and $P$. intermedia confused with it in the Altay region or Central Asia [5]. In comparison to morphological data, molecular data allow to differentiate more accurately these species, especially to distinguish $P$. anomala from $P$. intermedia and $P$. hybrida. In Paeonia anomala, several haplotypes within ycf1 gene have been found. We thus examined two molecular markers studied

* Corresponding author: $\underline{\text { degavi@ mail.ru }}$ 
previously, with a larger population sample, to elucidate more precisely intraspecific genetic diversity of Paeonia anomala.

\section{Experimental}

Plant material was collected during our expeditions to the Kola Peninsula, the Komi Republic and the Altai region or from herbaria ALTB, LE, and MW. To study intraspecific variability, 16 samples from different populations (Murmansk region, the Komi Republic, Altai region, Krasnoyarsk region, the Tyva Republic, Mongolia) were used.

Total genomic DNA was isolated from leaf tissue using the NucleoSpin Plant isolation kit (Macherey-Nagel, Düren, Germany) following the manufacturer's instructions. To amplify the internal transcribed spacers (ITS) of nrDNA, a pair of primers L and 4 was used [6]. The ycflbF and ycflbR primers were used to amplify the ycfl plastid gene fragment [7]. The PCR products were purified using a DNA Cleanup Mini kit (Evrogen, Moscow, Russia). Direct sequencing was performed on an ABI PRISM 3100 Genetic Analyzer (Applied Biosystems, Foster City, CA, USA) using the ABI Prism BigDye Terminator Cycle Sequencing Ready Reaction Kit for cycle sequencing reactions, following manufacturer's instructions. Given the relatively low signal and resolution from the nuclear marker, for plastid data alone we performed a principal coordinates analysis implemented in the program PAST 3.14 [8] to visualize patterns of variation. Gower general similarity coefficients were used.

\section{Results and discussion}

Paeonia anomala with its wide distribution range represents a most successful species within the genus. The species is usually preferred relatively moist habitats, growing in forest or rarely in bushes or meadows [3]. In the field, $P$. anomala reproduces only by seeds after cross-pollination. Its abundance through its distribution range can vary. For example, in its north-western range margin (the Kola Peninsula), the species is locally rare, with disjunct populations.

One of the reasons for such success may be the hybrid origin of the species, since the combination of diverged genomes allowed hybrid species to establish in novel environments [9]. It was recently concluded that $P$. anomala arose from crossing between Chinese $P$. veitchii and $P$. lactiflora [10]. Plastid data support that $P$. anomala is more similar to $P$. lactiflora than to $P$. veitchii. The modern distribution of $P$. anomala does not overlap with its parents, $P$. veitchii and $P$. lactiflora, which are found in southern and eastern regions of China. The phenology of these regions is distinct, suggesting that a novel adaptation has most likely resulted from the hybridization [10].

Our analyses of molecular data revealed the genetic differentiation of Paeonia anomala. Nuclear ITS sequences are characterized by several polymorphic positions, many of which indicate the hybrid origin of the species. However, two polymorphic positions are not of hybrid origin and can be considered as apomorphic for the species as a whole or its populations in the Kola Peninsula.

Ycf1 showed three haplotypes (H1, H2, H3) characterized by two nucleotide substitutions (Fig. 1). Besides, haplotype H3 are more similar to P. lactiflora than haplotypes $\mathrm{H} 1$ and $\mathrm{H} 2$ and, therefore, represent a plesiomorphic condition.

All ycf1 haplotypes co-occur in the Altay region (Fig. 2). Wherein, haplotype H1 restricted to the Altay region. Haplotype $\mathrm{H} 2$ had a mainly northern and northwestern distribution and reached the Murmansk region. Haplotype H3 had a mainly eastern distribution reaching Mongolia. 


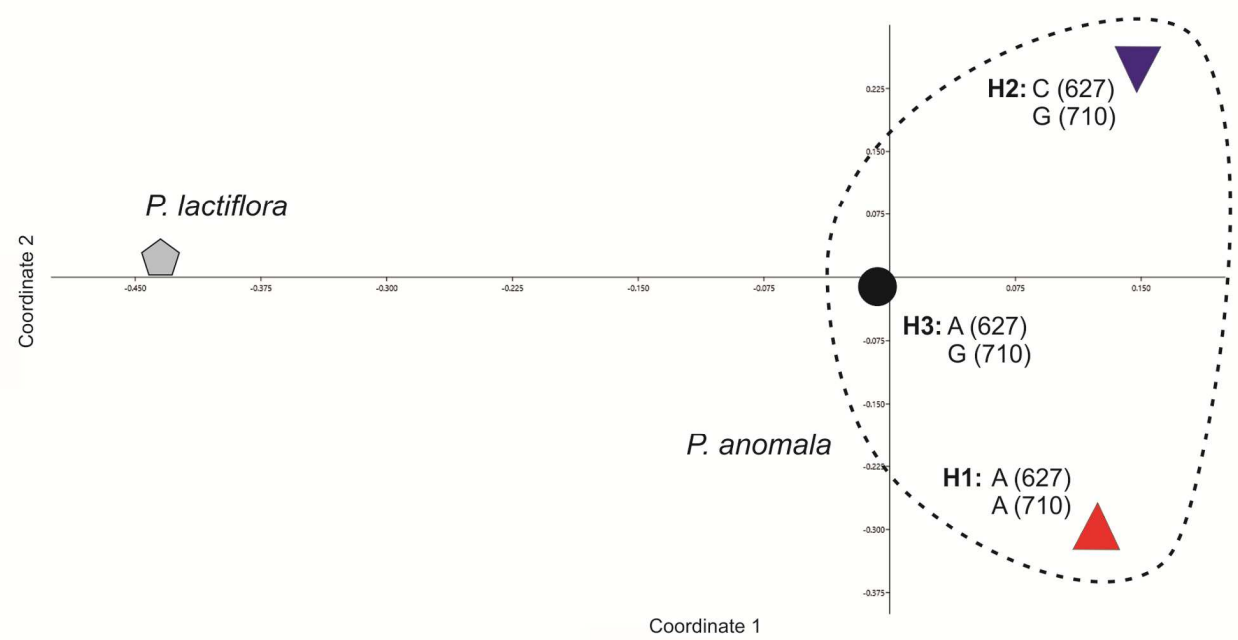

Fig. 1. Principal coordinates analysis of ycf1 sequence data for examined samples of Paeonia anomala. The three haplotype groups in ycf1 are coloured. Variable alignment positions are indicated.

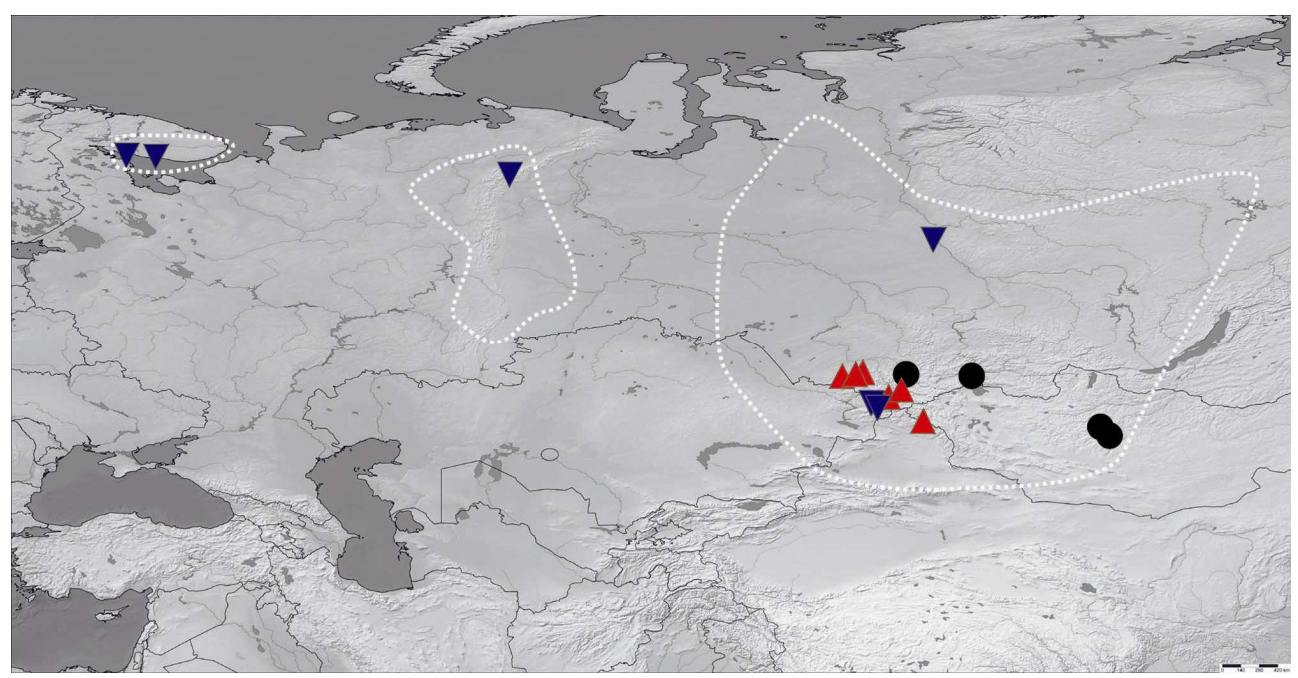

Fig. 2. Map showing locations of the 16 sampled populations of $P$. anomala with their ycf1 haplotypes. General distribution follows [3].

Our study of the genetic patterns of $P$. anomala populations, using nuclear and plastid markers, seems to be revealed a phylogeographic structure. The populations can be classified to differentiated genetic lines, and haplotypes are restricted to certain geographic regions.

Thus, our investigation of widely distributed and morphologically variable Paeonia anomala reveals a cryptic assemblage of genetically distinct taxa. Further investigation of the western part of its distribution range, with greater sampling will contribute to complete the picture of distribution direction.

We express our deep gratitude to the staff of the Yugyd Va National Park, the Kandalaksha Nature Reserve and the Katunsky Biosphere Reserve for their help in organizing our field research, as well as to the staff of the ALTB, LE and MW herbaria for their help in our work. The work is carried out as part of the Scientific Projects of the State 
Order of the Government of Russian Federation to Lomonosov Moscow State University (121031600193-7 and 121031600196-8).

\section{References}

1. T. Sang, D. J. Crawford, T. F. Stuessy, Proc. Natl. Acad. Sci. USA 92 (1995)

2. E. O. Punina, E. M. Machs, E. E. Krapivskaya, E. S. Kim, E. V. Mordak, Yu. A. Myakoshina, A. V. Rodionov, Russ. J. Genet. 48 (2012)

3. D. Y. Hong, Peonies of the World. Taxonomy and Phytogeography (Kew Publishing, London; Missouri Botanic Garden, St. Louis, 2010)

4. D. Y. Hong, K. Y. Pan, Ann. Missouri Bot. Gard. 91 (2004)

5. S. V. Efimov, G. V. Degtjareva, E. I. Terentieva, T. Kh. Samigullin, C. M. ValiejoRoman, The relationship of Paeonia anomala, P. intermedia, and P. hybrida (Paeoniaceae) species based on data on the ITS sequences of nuclear ribosomal DNA and ycf1 chloroplast DNA, Problems of Botany of South Siberia and Mongolia, Proceedings of the 15th International Scientific and Practical Conference, Barnaul, 2326 May (2016)

6. T. J. White, T. Bruns, S. Lee, J. Taylor J. Amplification and direct sequencing of fungal ribosomal RNA genes for phylogenetics // PCR protocols: a guide to methods and applications (Academic Press, New York, 1990)

7. W. Dong, C. Xu, C. Li, J. Sun, Y. Zuo, S. Shi, T. Cheng, J. Guo, S. Zhou, Sci. Rep. 5 (2015)

8. Ø. Hammer, D. A. T. Harper, P. D. Ryan, Palaeontol. Electron. 4 (2001)

9. L. H. Rieseberg, O. Raymond, D. M. Rosenthal, Z. Lai, K. Livingstone, T. Nakazato, J. L. Durphy, A. E. Schwarzbach, L. A. Donovan, C. Lexer, Science 301 (2003)

10. J. Pan, D. Zhang, T. Sang, Am. J. Bot. 94 (2007) 